\title{
Examination of Test Anxiety in Samples of Australian and U.S. Higher Education Students
}

\author{
Patricia A. Lowe ${ }^{1}$ \\ ${ }^{1}$ Department of Educational Psychology, University of Kansas, Lawrence, Kansas, USA \\ Correspondence: Patricia A. Lowe, Department of Educational Psychology, University of Kansas, Lawrence, KS, \\ 66045, USA. Tel: 1-785-864-9710. E-mail: tlowe@ku.edu
}

Received: August 6, 2019

Accepted: August 23, 2019 Online Published: September 7, 2019

doi:10.5539/hes.v9n4p33

URL: https://doi.org/10.5539/hes.v9n4p33

\begin{abstract}
Invariance testing and correlational analyses were conducted on a new test anxiety questionnaire, the Test Anxiety Measure for College Students (TAM-C), with 1.050 Australian and U.S. higher education students. The samples were administered the TAM-C along with other questionnaires. Results from the aforementioned analyses supported a modified six-factor structure for Australian students, U.S. students, males, and females and strong invariance across countries and across genders. Latent mean factor analyses found differences across countries and across genders on the questionnaire and validity evidence for the TAM-C scores in the Australian and U.S. student samples were found. Implications of the findings for counselors and researchers who work with Australian and U.S. undergraduates are discussed.
\end{abstract}

Keywords: test anxiety, Australian and U.S. higher education students, perfectionism, validity

\section{Introduction}

Test anxiety is a significant problem for students in institutions of higher learning (Knappe et al., 2011), including those students in higher education institutions in Australia (Mowbray, Boyle, \& Jacobs, 2015) and the United States (Tatum, Lundervold, \& Ament, 2006). Taylor (1956) conceptualized test anxiety as the anxiety students may experience when they take exams. Test anxiety creates a challenge for those who are test anxious, as students' educational studies may be negatively affected (Gerwing, Rash, Gerwing, Bramble, \& Landine, 2015; Mowbray et al., 2015) and degree completion may not be achieved (Gerwing et al., 2015).

Kavanagh, Ziin, and Mesagno (2016) have indicated that test anxiety research is needed in Australia because it has been an under studied area in this country. Kavanagh and colleagues have stated U.S. research findings could be used indirectly with the Australian student population if similar test anxiety results were found in Australia and the United States. Furthermore, psychometrically sound measures are needed to assess test anxiety in higher education students, including Australian postsecondary students (Mowbray et al., 2015).

\subsection{Existing Measures of Test Anxiety for Higher Education Students}

Extant measures are available to assess test anxiety in the higher education student population and most of these instruments include multiple dimensions. The Test Anxiety Inventory (TAI; Spielberger, 1980a) consists of two dimensions, a worry dimension and an emotionality dimension, but it is an old measure (Lowe \& Lee, 2008a). The TAI is almost 40 years old. Old measures are problematic as they have old norms and old items. Other old, multidimensional measures include the Reactions to Tests (RTT; Sarason, 1984) and the Test Anxiety Inventory-German version (TAI-G; Hodapp, Laux, \& Spielberger, 1982; Hodapp, 1991). Besides being old measures, Cassady and Johnson (2002) indicated that the TAI and RTT have limited item content assessing students' test anxiety before tests (Cassady \& Johnson, 2002). Rafferty, Smith, and Ptacek's (1997) research highlights the importance of including items that not only assess students' test anxiety during an exam, but also after as well as before an exam. The Cognitive Test Anxiety Scale (CTAS; Cassady \& Johnson, 2002), another measure of test anxiety, has some measurement problems involving some of its items that are reverse-coded (Thomas, Cassady, \& Heller, 2017). In 2015, the CTAS was updated and named the Cognitive Test Anxiety Scale-Revised (CTAR; Cassady \& Finch, 2015). The CTAR assesses only cognitive test anxiety. Furthermore, Alpert and Haber (1960) believed anxiety consists of two dimensions, a facilitating anxiety dimension and a debilitating (test) anxiety dimension. Students with facilitating anxiety experience some anxiety, but the anxiety 
they experience facilitates rather than interferes negatively with their test performance. None of the measures mentioned above include a facilitating anxiety dimension. Therefore, a new test anxiety measure is needed that (a) is more than one dimension, (b) was scaled only on the item ratings of higher education students, (c) includes items that assess anxiety students experience during different time periods in relation to a test, and (d) has a facilitating anxiety dimension (Lowe, 2018a).

\subsection{The New Measure of Test Anxiety}

A new questionnaire was published in 2018 for college and university students who experience test anxiety. The Test Anxiety Measure for College Students addresses the needs of researchers in the field of test anxiety as well as clinicians. Besides the inclusion of a facilitating anxiety scale, the TAM-C questionnaire consists of three cognitive scales of test anxiety, a behavioral scale of test anxiety, and a physical test anxiety scale (Lowe, 2018a). The test anxiety construct is believed to consist of many different dimensions and researchers need to identify those different dimensions and include those different dimensions in measures assessing the test anxiety construct (Zeidner, 1998). Moreover, the TAM-C questionnaire was designed specifically for students, ages 18 to 26, attending higher education institutions (Lowe, 2018a). The TAM-C measure does not assess test anxiety in students in grades 1-12 or adults, as the contexts in which these different age groups experience test anxiety are likely to be different. Moreover, the questionnaire assesses anxiety during different time periods in relation to when students take exams. Test anxiety may occur during the time students actually take an exam, but it may also occur after an exam or possibly before an exam. Furthermore, the questionnaire was factor analyzed with only the item ratings of higher education students, resulting in a six-factor solution (Lowe, 2018a). However, it is unknown if the TAM-C six-factor structure would be found in an Australian sample of higher education students.

\subsection{Cultural Contexts and Australia and the United States}

Culture is thought to have some bearing on test anxiety (Lowe et al., 2008b). Australia and the United States are reported to have similar cultural values (Ollendick, King, \& Frary, 1989) and socialization practices (Russell, Hart, Robinson, \& Olsen, 2003). However, differences do exist in their educational systems and organization (Kavanagh et al., 2016; Triventi, 2014). Kavanagh and colleagues (2016) asserted that U.S. students take examinations and are required to perform well on these tests during their undergraduate years in order to gain entry into U.S. graduate programs and therefore, they may experience more test anxiety than Australian students who must apply and be interviewed in order to gain entry into Australian graduate programs. Triventi (2014) examined higher educational systems in 16 different countries, including Australia and the United States. Triventi found North American educational systems, which included the United States, have higher accountability to outside agencies in comparison to Anglo-Saxon educational systems, which included Australia. Educational institutions with higher accountability may increase their testing of students in order to report student outcomes to external agencies and the increased testing may result in more test anxiety for students who attend these educational institutions (Lowe, 2019a).

Only one published study to the author's knowledge has examined differences in test anxiety between Australian and U.S. post-secondary students. Kavanagh et al.'s (2016) study included a small sample (89 Australian and 143 U.S. students) and explored test anxiety differences on the TAI. In their study, however, Kavanagh and colleagues did not test for invariance across Australian and U.S. students before exploring group differences.

\subsection{Gender and Test Anxiety}

Hembree's (1988) meta-analysis found that males in grades 11-12 and postsecondary school reported less test anxiety than females. Hembree also found that these gender differences tend to decline during the high school and postsecondary years. Differences across gender have also been reported in facilitating anxiety in some studies, with females reporting lower levels of facilitating anxiety than males (Hembree, 1988); however, in more recent studies, differences in facilitating anxiety between genders have not been found (Lowe, 2018a; Lowe \& Lee, 2008a).

\subsection{Relationships between Test Anxiety and Other Constructs}

Relationships between another test anxiety measure and a perfectionism measure and the TAM-C were examined in the current study. Concurrent validity evidence was examined with another test anxiety measure. Researchers have reported correlations in the medium to large range between scores of measures of test anxiety (Lowe, 2018a; Spielberger, 1980b). In addition, convergent validity evidence was examined with a perfectionism measure, and more specifically, a perfectionism measure that includes a scale assessing socially prescribed (self-critical) perfectionism. Socially prescribed perfectionism has been positively linked to negative traits (Hewitt \& Flett, 2004), such as test anxiety (Mills \& Blankstein, 2000; Stöeber, Feast, \& Hayward, 2009). In contrast, 
self-oriented (i.e., rigid) perfectionism, another major type of perfectionism, has been positively linked to negative and positive traits (Hewitt \& Flett, 2004). Researchers have hypothesized a relationship between perfectionism and test anxiety in which perfectionism is believed to be a risk factor in students' anxiety (Egan, Wade, \& Shafran, 2011), with Damian, Negru-Subtirica, Stöeber, and Băban's (2017) recent work providing empirical support for perfectionism concerns (socially prescribed perfectionism) as a risk factor in older adolescents' anxiety. Damian and colleagues found perfectionism concerns but not perfectionism strivings (self-oriented perfectionism) predicted increases in anxiety symptoms for older adolescents over time. Furthermore, in Stöeber et al.'s (2009) study, the authors examined the relationship between socially prescribed perfectionism and different dimensions of test anxiety (i.e., emotionality, interference, lack of confidence, and worry) and they found small to medium, positive relations between socially prescribed perfectionism and test anxiety.

\subsection{Aims and Hypotheses for the Current Studies}

The present study included three aims. The first aim was to test for invariance on the TAM-C across Australian and U.S. students and male and female students. In order to make international comparisons between higher education students in different countries, it is important to determine whether the construct (e.g., test anxiety) is equivalent across groups. If equivalency is supported, group differences can be investigated. The author hypothesized that invariance across Australian students and U.S. students, and male students and female students would be found.

The second aim was to examine score differences related to Australia and U.S. higher education students, and male and female higher education students on the TAM-C. The author did not include a hypothesis about differences across Australian and U.S. higher education students because of the limited research conducted in Australia. As for differences across male and female higher education students, the author hypothesized that female higher education students would have more test anxiety than male higher education students and based on more recent research, no differences across male and female higher education students would be found in facilitating anxiety.

The third aim was to examine validity evidence of the TAM-C in the Australian and U.S. higher education student samples. The author hypothesized that positive, medium to large associations would be found between two test anxiety measures in the Australian and U.S. higher education student samples. In addition, the relationship between socially prescribed (self-critical) perfectionism and test anxiety was investigated. The author hypothesized that positive, small to medium associations would be found between the TAM-C test anxiety scales and self-critical perfectionism scales in the Australian and U.S. student samples.

\section{Method}

\subsection{Participants}

The participants included 1,050 students, 524 (49.9\%) males and 526 (50.1\%) females. For the 525 Australian student sample, $262(49.9 \%)$ were males and $263(50.1 \%)$ were females. The age range of the Australian students was 18 to $26(M=21.19, S D=2.38)$. One hundred and sixty-seven $(31.8 \%), 150(28.6 \%), 89(17.0 \%), 62$ $(11.8 \%)$, and $57(10.9 \%)$ students were in their first- through fifth-year in college, respectively. The ethnic distribution of the sample was Australian $(n=377,71.8 \%)$, European $(n=31,5.9 \%)$, Asian $(n=64,12.2 \%)$, Mixed/Multiracial $(n=36,6.9 \%)$, and other $(n=17,3.2 \%)$. Most common majors for the Australian students were Business (20.57\%), Allied Health Sciences (14.29\%), Social Sciences (9.14\%), Physical Sciences (8.38\%), and Engineering $(8.00 \%)$. All students attended higher education institutions in Australia and resided in the northern (11.4\%), eastern (36.0\%), western (17.1\%), and southern (32.2\%) regions of Australia, with $3.3 \%$ of the students not reporting a specific region.

The U.S. sample also consisted of 525 undergraduate students, 262 males and 263 females, with an age range of 18 to $26(M=21.51, S D=2.50)$. Year in college for the U.S. sample included $111(21.1 \%)$ in their first year, 155 (29.5\%) in their second year, $113(21.5 \%)$ in their third year, $98(18.7 \%)$ in their fourth year, and $48(9.1 \%)$ in their fifth year. Ethnic composition of the U.S. sample was White $(n=299,57.0 \%)$, Hispanic $(n=96,18.3 \%)$, Black/African American $(n=72,13.7 \%)$, Asian/Pacific Islander $(n=36,6.9 \%)$, Native American/Alaskan Native $(n=4, .8 \%)$, and other $(n=18,3.4 \%)$. Most common majors for the U.S. students were Business (25.9\%), Physical Sciences (23.0\%), Social Sciences (18.1\%), and Allied Health Sciences (9.9\%). All students attended U.S. higher education institutions and resided in the northern (14.7\%), eastern (20.2\%), midwestern (11.4\%), southern (29.5\%), and western (23.4\%) geographic areas of the United States. A small percentage (.8\%) did not indicate the geographic region were they resided. 


\subsection{Instruments}

The Big Three Perfectionism Scale, Short Form, the BTPS-SF, includes a Self-Critical (Socially Prescribed) Perfectionism scale, a Narcissistic (Other-Oriented) Perfectionism scale, and a Rigid (Self-Oriented) Perfectionism scale (Feher et al., under review). The Self-Critical Perfectionism scale assesses individuals' perceptions that others demand individuals to be faultless and the Rigid Perfectionism scale measures individuals' perceptions that they must do everything correctly. The Narcissistic Perfectionism scale assesses individuals' perceptions that others should be faultless. Individuals respond to the BTPS-SF items on a 5-point scale, with anchor descriptors ranging from strongly disagree to strongly agree (Feher et al., under review). Cronbach's alphas for the perfectionism scores ranged from .86 to .87 for the Australian sample and .85 to .88 for the U.S. sample in the current study.

The TAI assesses test anxiety in 9-12 grade students and adults. The TAI has a 4-point response scale, with anchor descriptors of almost never and almost always (Spielberger, 1980b). Cronbach's alphas for the TAI scores were .93 for the Australian higher education sample and .92 for the U.S. higher education sample in the present study.

The TAM-C measures test anxiety and facilitating anxiety. The five test anxiety scales are Physiological Hyperarousal (7 items; physical-related symptoms related to test anxiety), Cognitive Interference ( 7 items; self-critical thoughts that distract the self from focusing on tests), Task Irrelevant Behaviors (6 items; occurrence of avoidance and jittery behaviors in evaluative situations), Worry (8 items; concerns about failing tests), and Social Concerns ( 7 items; concerns about others' negative reactions for one's possible poor test performance) scales. The sixth scale is the Facilitating Anxiety scale. This scale includes 7items and measures the slight nervousness felt in exam situations that improves one's test performance. The TAM-C has a 4-point response scale, with anchor descriptors of never and almost always (Lowe, 2018a). Latent reliabilities of .78 to .94 for the six scales were found in samples of U.S. and Canadian higher education students (Lowe, 2019b).

\subsection{Procedures}

Approval was acquired from the research review board where the author is employed prior to beginning the study. Participants were recruited by a U.S. survey company named Qualtrics. The Australian and U.S. higher education students gave their consent before they filled out the measures online. The students took the survey in approximately 15 minutes.

\subsection{Data Analysis}

The demographics of the sample were analyzed using SPSS, Version 22 (IBM, 2013). Following the computation of the descriptive statistics, CFAs were performed with Mplus 7.11 (L. K. Muthén \& B. O. Muthén, 1998-2013) software. CFAs were performed with the responses of Australian males, Australian females, U.S. males, U.S. females, males, females, Australian students, and U.S. students on the measure to determine whether the model with six first-order factors was an acceptable or very good model fit for each of these groups on the TAM-C. Little's (2013) suggested guidelines were used to indicate a poor, mediocre, acceptable, and very good model fit for the data. A poor model fit was a root mean square error of approximation (RMSEA) value of $>.10$ and a Tucker-Lewis index (TLI)/Non-normed fit index (NNFI) value and a comparative fit index (CFI) value of $<.85$, a mediocre model fit was a RMSEA value of $\leq .10$ and a TLI/NNFI value and a CFI value of $\leq .90$, an acceptable model fit was a RMSEA value of $\leq .08$, and a TLI/NNFI value and a CFI value of $>.90$, and a very good model fit was a RMSEA value of $<.05$, and a TLI/NNFI value and a CFI value of $>.95$. For the CFAs and multi-group CFAs to follow, the weighted least squares means and variance adjusted (WLSMV) estimation was used to estimate the parameters.

After the CFAs were performed, multi-group CFAs followed. The four multi-group comparisons were between Australian and U.S. students; Australian males and Australian females; U.S. males and U.S. females; males and females also using Mplus 7.11 software to examine the equivalence of the test anxiety construct across country and across gender. Configural, weak, and strong invariance were conducted for each of the four multi-group comparisons. Chen's (2007) criteria, a change in the CFI of $\leq .-01$ and a change in the RMSEA > .015, were used to indicate whether configural, weak, and strong invariance was supported across groups.

The four multi-group comparison results would dictate whether country and gender differences would be examined. If strong invariance was supported for the four multi-group comparisons, then latent mean analyses would be performed to examine differences across countries and across genders on the TAM-C. Byrne, Shavelson, and Muthén (1989) have indicated that strong invariance is needed in order to investigate group differences. 
Finally, Pearson Product Moment Correlation Coefficients were computed between the TAM-C test anxiety and facilitating anxiety scores and the scores of the TAI and the TBPS-SF in the Australian and U.S. samples. Guidelines used to indicate a Pearson $\mathrm{r}$ correlation was small was .10, medium .30, and large .50 (Green \& Salkind, 2014).

\section{Results}

\subsection{Examination of the TAM-C Six-Factor Structure}

CFAs were performed to determine whether the model with six factors would provide an acceptable or very good model fit for the responses of the different groups on the TAM-C. The CFA results suggested a correlated error between two items. Based on these results, the model with six factors was revised to include a correlated error between two Social Concerns items (i.e., Items 5 and 15) for each of the groups and the CFAs were run again. Results of the modified model with six factors for each of the groups are shown in Table 1. The modified model with six factors provided an acceptable model fit for each of the groups, according to Little's (2013) suggested guidelines.

Table 1. Fit Indices for the Modified Six-Factor Model and Latent Reliability Ranges for Australian and U.S. Students, and Males and Females

\begin{tabular}{lllllll}
\hline Group & Latent Reliability Ranges & WLSMV $\chi^{2}$ & df & RMSEA[90\% CI] & TLI/NNFI & CFI \\
\hline Australian Males & $.802-.927$ & $1466.208^{* * * *}$ & 844 & $.053[.048, .058]$ & .928 & .932 \\
Australian Females & $.829-.946$ & $1456.400^{* * * *}$ & 844 & $.053[.048, .057]$ & .949 & .953 \\
U.S. Males & $.819-.926$ & $1522.640^{* * * *}$ & 844 & $.055[.051, .060]$ & .920 & .925 \\
U.S. Females & $.806-.936$ & $1451.464 * * * *$ & 844 & $.052[.048, .057]$ & .934 & .939 \\
Males & $.810-.925$ & $2222.036 * * * *$ & 844 & $.056[.053, .059]$ & .920 & .925 \\
Females & $.815-.938$ & $2068.154^{* * * *}$ & 844 & $.053[.050, .055]$ & .942 & .946 \\
Australian Students & $.811-.934$ & $2150.273^{* * * *}$ & 844 & $.054[.051, .057]$ & .936 & .940 \\
U.S. Students & $.808-.930$ & $2161.744 * * * *$ & 844 & $.055[.052, .057]$ & .928 & .932 \\
\hline
\end{tabular}

Note. WLSMV $\chi^{2}=$ robust mean-and-variance adjusted chi square; $\mathrm{df}=$ degrees of freedom; RMSEA = root mean square error of approximation; $90 \% \mathrm{CI}=90 \%$ confidence interval; TLI/NNFI = Tucker-Lewis Index/Non-normed fit index; CFI = comparative fit index; $p$ $<.0001$

\subsection{Latent Reliabilities for the TAM-C Scales}

Composite reliabilities were calculated instead of Cronbach alphas for the TAM-C scales for each of the groups because equivalency of the factor loadings could not be assumed (Yang \& Green, 2011). The latent reliabilities for Australian students, U.S. students, Australian males, Australian females, U.S. males, U.S. females, males, and females on each of the TAM-C six scales ranged from .802 to .946 (see Table 1).

\subsection{Invariance across Gender and Country}

Tests of invariance followed between Australian and U.S. higher education students, Australian male students and Australian female students, U.S. male students and U.S. female students, and male students and female students. The findings of the multi-group comparison supported strong invariance for each comparisons made (see Table 2). All changes in the CFIs and RMSEAs were within Chen's (2007) criteria. 
Table 2. Results of Measurement Invariance Across Country and Gender

\begin{tabular}{|c|c|c|c|c|c|c|c|c|c|}
\hline Groups & WLSMV $\chi 2$ & $d f$ & $\Delta \mathrm{WLSMV}^{2}$ & $\Delta d f$ & RMSEA $[90 \% \mathrm{CI}]$ & $\triangle$ RMSEA & TLI/NNFI & CFI & $\Delta \mathrm{CFI}$ \\
\hline \multicolumn{10}{|l|}{ Australia and } \\
\hline \multicolumn{10}{|l|}{ United States } \\
\hline 1. Configural & $4311.225 * * * *$ & 1688 & & & $.054[.052, .056]$ & & .932 & .937 & \\
\hline 2. Weak & $4009.562 * * * *$ & 1725 & $58.568 *$ & 37 & $.050[.048, .052]$ & .004 & .942 & .945 & .008 \\
\hline 3. Strong & $4321.361 * * * *$ & 1848 & $331.171 * *$ & 123 & $.050[.048, .052]$ & .000 & .944 & .943 & .002 \\
\hline \multicolumn{10}{|c|}{ Australian Males } \\
\hline \multicolumn{10}{|c|}{ and Females } \\
\hline 1. Configural & $2922.645^{* * * * *}$ & 1688 & & & $.053[.050, .056]$ & & .941 & .944 & \\
\hline 2. Weak & $2896.556^{* * * * *}$ & 1725 & $80.564 * *$ & 37 & $.051[.048-.054]$ & .002 & .945 & .947 & .003 \\
\hline 3. Strong & $3091.905 * * * *$ & 1848 & $309.715^{* *}$ & 123 & $.051[.048, .058]$ & .000 & .945 & .944 & .003 \\
\hline \multicolumn{10}{|c|}{ U.S. Males and Females } \\
\hline 1. Configural & $2975.028 * * * *$ & 1688 & & & $.054[.051, .057]$ & & .927 & .932 & \\
\hline 2. Weak & $2849.474 * * * *$ & 1725 & 42.693 & 37 & $.050[.047, .053]$ & .004 & .938 & .941 & .009 \\
\hline 3. Strong & $3002.353^{* * * * *}$ & 1848 & $215.482 * *$ & 123 & $.049[.046, .052]$ & .001 & .941 & .939 & .002 \\
\hline \multicolumn{10}{|c|}{ Males and Females } \\
\hline 1. Configural & $4290.203 * * * *$ & 1688 & & & $.054[.052, .056]$ & & .933 & .937 & \\
\hline 2. Weak & 4057.830 ***** & 1725 & $83.836^{* *}$ & 37 & $.051[.049, .053]$ & .003 & .941 & .943 & .006 \\
\hline 3. Strong & $4290.830 * * * *$ & 1848 & $354.260^{* *}$ & 123 & $.050[.048-.052]$ & .001 & .942 & .941 & .002 \\
\hline
\end{tabular}

Note. $\mathrm{WLSMV} \chi 2=$ robust mean-and-variance adjusted chi-square; $\triangle \mathrm{WLSMV} \chi 2=$ change in the robust mean-and-variance adjusted chi-square; $d f=$ degrees of freedom; $\Delta d f=$ change in degrees of freedom; RMSEA = root mean square error of approximation; $90 \% \mathrm{CI}=90 \%$ confidence interval; TLI/NNFI = Tucker-Lewis Index/Non-normed fit index; CFI = comparative fit index; $\Delta \mathrm{CFI}=$ change in the comparative fit index; * $p<.05 ; * * p<.01, * * * * p<.0001$

\subsection{Score Differences Related to Country and Gender}

Score differences related to country and gender were examined on the TAM-C through latent mean analyses. Australian higher education students reported a higher level of physiological hyperarousal $(\beta=-.321, S E=.069$, $p<.001)$ than U.S. higher education students, and female students endorsed higher levels of physiological hyperarousal $(\beta=.157, S E=.065, p<.05)$ and worry $(\beta=.396, S E=.061, p<.001)$ than male students. Australian females reported higher levels of cognitive interference $(\beta=.176, S E=.087, p<.05)$, physiological hyperarousal $(\beta=.247, S E=.090, p<.01)$, and worry $(\beta=.428, S E=.083, p<.001)$, than Australian males, and U.S. females endorsed a higher level of worry $(\beta=.361, S E=.089, p<.001)$ than U.S. males.

\subsection{External Validity Evidence}

Pearson Product Moment Correlation Coefficients were computed between the TAM-C scores and the scores of the TAI and BTPS-SF (see Table 3). Correlations were large between the test anxiety scores of the TAM-C and the TAI scores in both the Australian and U.S. samples. Moreover, three of the TAM-C test anxiety (Physiological Hyperarousal, Task Irrelevant Behaviors, and Cognitive Interference) scores had medium correlations with the TBPS-SF Self Critical Perfectionism scores in the Australian sample. However, two of the TAM-C test anxiety (Social Concerns and Worry) scores had large correlations with the TBPS-SF Self Critical Perfectionism scores in the Australian sample. For the U.S. sample, the majority of correlations between the TAM-C test anxiety scores and the TBPS-SF Self-Critical Perfectionism scores were in the medium range, with the exception of the correlation between the TAM-C Task Irrelevant Behaviors scores and the TBPS-SF Self-Critical Perfectionism scores, which was small. Although the large correlations between the TAM-C Worry and Social Concerns scores and the TBPS Self-Critical Perfectionism scores in the Australian sample were somewhat unexpected. Overall, the results lend support for the validity evidence for the TAM-C test anxiety scores in both the Australian and U.S. samples. 
Table 3. Correlations between the Test Anxiety Measure for College Students (TAM-C) Scores and Scores of the Test Anxiety Inventory (TAI), and the Three Big Perfectionism Scale-Short Form (TBPS-SF) in the Australian and U.S. Samples

\begin{tabular}{lllllll}
\hline Scale & \multicolumn{6}{l}{ TAM-C } \\
\cline { 2 - 6 } & 1 & 2 & 3 & 4 & 5 & 6 \\
\hline \multirow{2}{*}{ TAI Total } & $.78^{* *}$ & $.57^{* *}$ & $.70^{* *}$ & $.77^{* *}$ & $.65^{* *}$ & $.14^{* *}$ \\
& $\left(.67^{* *}\right)$ & $\left(.57^{* *}\right)$ & $\left(.67^{* *}\right)$ & $\left(.73^{* *}\right)$ & $\left(.61^{* *}\right)$ & $\left(.27^{* *}\right)$
\end{tabular}

TBPS-SF

\begin{tabular}{lllllll} 
Rigid Perfectionism & $.23^{* *}$ & .08 & .06 & $.36^{* *}$ & $.34^{* *}$ & $.23^{* *}$ \\
& $\left(.19^{* *}\right)$ & $\left(.17^{* *}\right)$ & $\left(.14^{* *}\right)$ & $\left(.29^{* *}\right)$ & $\left(.33^{* *}\right)$ & $\left(.27^{* *}\right)$ \\
Self-Critical Perfectionism & $.48^{* *}$ & $.32^{* *}$ & $.37^{* *}$ & $.61^{* *}$ & $.54^{* *}$ & $.13^{* *}$ \\
& $\left(.35^{* *}\right)$ & $\left(.29^{* *}\right)$ & $\left(.33^{* *}\right)$ & $\left(.48^{* *}\right)$ & $\left(.47^{* *}\right)$ & $\left(.20^{* *}\right)$ \\
\multirow{2}{*}{ Narcissistic Perfectionism } & $.23^{* *}$ & $.16^{* *}$ & $.14^{* *}$ & -.01 & $.21^{* *}$ & $.13^{* *}$ \\
& $\left(.29^{* *}\right)$ & $\left(.19^{* *}\right)$ & $\left(.16^{* *}\right)$ & $(.04)$ & $\left(.28^{* *}\right)$ & $\left(.25^{* *}\right)$
\end{tabular}

Note. 1 = Physiological Hyperarousal; 2 = Task Irrelevant Behaviors; 3 = Cognitive Interference; 4 = Worry; $5=$ Social Concerns; and $6=$ Facilitating Anxiety; U.S. higher education student correlations are in parentheses; ${ }^{*} p<.05$, ${ }^{* *} p<.01$

\section{Discussion and Conclusion}

The study's first goal was to examine invariance across country and across gender on the TAM-C. The results from the current study supported strong invariance of the TAM-C scores across country and gender, suggesting that the test anxiety construct, as measured by the TAM-C, is equivalent across the two nations and genders, and researchers and clinicians can interpret the scores for Australian and U.S. higher education students the same on the measure. This is the first published study to the author's knowledge to conduct an international comparison between Australian and U.S. post-secondary students on a test anxiety measure using measurement invariance.

The study's second goal was to examine differences across countries and across genders on the TAM-C in samples of Australian and U.S. post-secondary students. Examination of group differences found U.S. higher education students reported less test anxiety (i.e., physiological hyperarousal test anxiety) than Australian higher education students. Kavanagh and colleagues (2016) suggested U.S. higher educational students may get used to the additional testing they encounter in their educational experiences, resulting in less test anxiety experienced for this group of students in comparison to Australian higher education students. This explanation may help account for the difference in physiological hyperarousal symptoms reported across countries.

Females were found to report more worry and physical symptoms than males. In addition, Australian females reported more worry, physiological hyperarousal, and cognitive interference symptoms than Australian males, and U.S. females reported more worry symptoms than U.S. males. These findings are in agreement with the results reported in the test anxiety literature (Hembree, 1988). Moreover, no differences across gender were found on the TAM-C Facilitating Anxiety scale. These results are similar to more recent research with U.S. post-secondary students (Lowe, 2018a). Overall, the findings of differences across countries and across genders make a significant contribution to the literature, as a dearth of studies have been conducted in the area of test anxiety in higher educational settings in Australia where test anxiety is viewed as a pervasive problem (Mowbray et al., 2015).

The study's third goal was to examine validity evidence for the TAM-C scores in samples of Australian and U.S. students. The Pearson $r$ correlations between the two test anxiety measures' scores were large. These findings are similar to Lowe's $(2018 \mathrm{a}, \mathrm{b})$ studies with Canadian and U.S. post-secondary students where medium to large, positive correlations were found between the TAM-C scores and scores on another measure that includes a test anxiety scale.

Relations between self-critical perfectionism and test anxiety were found to be in the medium to large range in the Australian sample and small to medium range for the U.S. sample. These findings partly support the hypothesized small to medium correlations between these variables and are similar to Stöber et al.'s (2009) findings reported with a sample of British higher education students. Besides the small to medium correlations found, large correlations were reported between the TAM-C Worry and Social Concerns scores and the TBPS-SF 
Self-Critical Perfectionism scores in the Australian sample. These findings were somewhat unexpected, but not completely surprising, as worry, social concerns, and self-critical perfectionism items assess somewhat similar content. More specifically, worry and self-critical perfectionism items assess failure, with worry measuring an individual's fear of failure in testing situations (Lowe, 2018a) and self-critical perfectionism assessing one's disappointment to perceived failure (Dunkley, Zuroff, \& Blankstein, 2003; Feher et. al., under review). Moreover, social concerns and self-critical perfectionism items assess concerns about the self in relation to others, with social concerns measuring excessive concerns an individual has about the negative reactions of others regarding possible poor test performance (Lowe, 2018a) and self-critical perfectionism measuring excessive concerns a person has about the perceived demands from others for one to be perfect (Hewitt \& Flett, 1991). This is one explanation proffered for the large correlations reported in the Australian sample. However, another possibility is that culture may have influenced in some way the relationship between social concerns and self-critical perfectionism and between worry and self-critical perfectionism in the Australian sample. Future research is needed to explore this issue in more detail. In either case, the findings reported provide evidence supporting the validity of the scores of the TAM-C in an Australian and a U.S. student sample.

Small correlations were reported between the TAM-C Physiological Hyperarousal scores and the TBPS-SF Rigid Perfectionism scores in the Australian and U.S. samples. These findings are similar to the results found in Stöeber and colleagues' (2009) study with British higher education students. Moreover, a small correlation and a medium correlation was found between the TAM-C Worry scores and TBPS-SF Rigid Perfectionism scores in the U.S. and Australian samples, respectively. The magnitude of these correlations are not substantially different from each other and these findings are also similar to the medium correlation found in Stöeber and colleagues' (2009) study. In addition, medium correlations were found between the TAM-C Social Concerns scores and the TBPS-SF Rigid Perfectionism scores in both the Australian and U.S. samples. In contrast, negligible relationships were reported between the TAM-C Cognitive Interference and Task Irrelevant Behaviors scores and TBPS-SF Rigid Perfectionism scores in the Australian sample and small correlations were found between the TAM-C Cognitive Interference and Task Irrelevant Behaviors scores and TBPS-SF Rigid Perfectionism scores in the U.S. sample. It is possible that some self-oriented perfectionists in the Australian sample may have high expectations for themselves and they work on meeting those expectations by using adaptive strategies (see Stöeber et al., 2009), such as persistence, remaining focused, and self-efficacy (Hewitt \& Flett, 1991) and perhaps, these individuals are less likely to experience test anxiety in the form of task-irrelevant (i.e., avoidant) behaviors and cognitive interference (i.e., distracting thoughts). This explanation may account for the two nonsignificant correlations reported between the TAM-C Task-Irrelevant Behaviors and Cognitive Interference scores and the TBPS-SF Rigid Perfectionism scores in the Australian sample in the current study. Overall, this is the first study to examine the relationships between self-oriented (rigid) and socially prescribed (self-critical) perfectionism and task-irrelevant behaviors test anxiety and social concerns test anxiety.

Research has not investigated the relationship between the different dimensions of test anxiety and narcissistic perfectionism among post-secondary students. Small, positive correlations were found between the TAM-C test anxiety scores and the TBPS-SF Narcissistic Perfectionism scores, with the exception of a negligible correlation between the TAM-C Worry scores and the TBPS-SF Narcissistic Perfectionism scores in both the Australian and the U.S. samples. The negligible correlations between worry and narcissistic perfectionism in both the Australian and U.S. samples may be due to narcissistic perfectionists may be more likely to blame others for their imperfections (see Hewitt \& Flett, 1991; Onwuegbuzie \& Daley, 1999) rather than worrying about one's own perceived failures. Similarly, small, positive correlations were found between facilitating anxiety and different types of perfectionism. These findings between facilitating anxiety and different types of perfectionism with postsecondary students are the first to be reported.

Limitations of the study include a larger percentage of first-year Australian higher education students in comparison to the percentage of U.S. post-secondary students. In addition, the percentages of third- and fourth-year U.S. higher education students was larger than the percentages of third- and fourth-year Australian higher education students. Moreover, there was a somewhat larger percentage of Asians (12.2\%) in the Australian sample compared to the actual Australian population (approximately 8.4\%; Australian Bureau of Statistics, 2016). Replication of the present study should be conducted in the future with a sample of Australian and U.S. post-secondary students that better mirrors the ethnicity of the Australian population and includes equal numbers of students at each grade level from both countries.

The findings have important implications for counselors and researchers who work with Australian and U.S. higher education students. First, Australian researchers can use U.S. test anxiety results and apply these findings indirectly to Australian students "without relying on unwarranted assumptions" (Kavanagh et al., 2016, p. 556). 
Second, Australian higher education students are more likely to experience physiological hyperarousal symptoms associated with evaluative situations than U.S. higher education students. This is the first published study to find test anxiety differences between Australian and U.S. higher education students to the author's knowledge. Thus, strategies, such as relaxation training (Reiss et al, 2017; Zeidner, 1998), may be helpful to use with test-anxious Australian students, to reduce their physical symptoms associated with test anxiety. A third implication is that counselors need to be cognizant that females reported more test anxiety than males; however, males may also experience test anxiety. Counselors who work with Australian and U.S. male and female students who report academic difficulties should assess these students for test anxiety and if students are found to be test-anxious, then counselors should recommend appropriate interventions based on students' symptom presentation (Lowe, 2019a). Based on the findings reported in the current study, the TAM-C has the potential to be a solid, psychometrically-sound questionnaire that can be used with Australian and U.S. post-secondary students.

\section{Acknowledgments}

This work was supported by the University of Kansas under Grants 2338000-906CFI and 2338707-906.

\section{References}

Alpert, R., \& Haber, R. N. (1960). Anxiety in academic achievement situations. Journal of Abnormal and Social Psychology, 61, 207-215. https://doi.org/10.1037/h0045464

Australian Bureau of Statistics. (2016). Cultural diversity in Australia, 2016. Retrieved from http://www.abs.gov.au

Byrne, B. M., Shavelson, R. J., \& Muthen, B. (1989). Testing for the equivalence of factor covariance and mean structures: The issue of partial measurement invariance. Psychological Bulletin, 105, 456-466. https://doi.org/10.1037/0033-2909.105.3.456

Cassady, J. C., \& Finch, W. F. (2015). Using factor mixture modeling to identify dimensions of cognitive test anxiety. Learning and Individual Differences, 41, 14-20. https://doi.org/10.1016/j.lindif.2015.06.002

Cassady, J. C., \& Johnson, R. E. (2002). Contemporary Educational Psychology, 27, 270-295. https://doi.org/10.1006/ceps.2001.1094

Chen, F. F. (2007). Sensitivity of goodness of fit indexes to lack of measurement invariance. Structural Equation Modeling, 14, 464-504. https://doi.org/10.1080/10705510701301834

Damien, L. E., Negru-Subtirica, O., Stöeber, J., \& Băban, A. (2017). Perfectionistic concerns predict increases in adolescents' anxiety symptoms: A three-wave longitudinal study. Anxiety, Stress, \& Coping, 30, 551-561. https://doi.org/10.1080/10615806.2016.1271877

Dunkley, D. M., Zuroff, D. C., \& Blankstein, K. R. (2003). Self-critical perfectionism and daily affect: Dispositional and situational influences on stress and coping. Journal of Personality and Social Psychology, 84, 234. https://doi.org/10.1037/0022-3514.84.1.234

Egan, S. J., Wade, T. D., \& Shafran, R. (2011). Perfectionism as a transdiagnostic process: A clinical review. Clinical Psychology Review, 31, 203-212. https://doi.org/10.1016/j.cpr.2010.04.009

Feher, A., Smith, M. M., Saklofske, D. H., Plouffe, R. A., Wilson, C. A., \& Sherry, S. B. (under review). The Big Three Perfectionism Scale-Short Form (BTPS-SF): Development of a brief 16-item self-report measure of multidimensional perfectionism.

Gerwing, T. G., Rash, J. A., Gerwing, A. M. A., Bramble, B., \& Landine, J. (2015). Perceptions and incidence of test anxiety. The Canadian Journal for Scholarship of Teaching and Learning, 6(3), Article 3. https://doi.org/10.5206/cjsotl-rcacea.2015.3.3

Green, S. B., \& Salkind, N. J. (2014). Using SPSS for Windows and MacIntosh $\left(7^{\text {th }}\right.$ ed.). Upper Saddle River, NJ: Pearson.

Hembree, R. (1988). Correlates, causes, effects, and treatment of TA. Review of Educational Research, 58, 47-77. https://doi.org/10.2307/1170348

Hewitt, P. L., \& Flett, G. L. (1991). Perfectionism in the self and social contexts: Conceptualization, assessment, and association with psychopathology. Journal of Personality and Social Psychology, 60, 456-470. https://doi.org/10.1037/0022-3514.60.3.456

Hewitt, P. L., \& Flett, G. L. (2004). Multidimensional perfectionism scale; Technical manual. Toronto, Canada: Multi-Health Systems. 
Hodapp, V. (1991). Das Prüfungsängstlichkeitsinventar TAI-G: Eine erweiterte und modifizierte version mit vier komponenten [The Test Anxiety Inventory TAI-G: An expanded and modified version with four components]. Zeitschrift für Pädagogische Psychologie, 5(2), 121-130.

Hodapp, V., Laux, L., \& Spielberger, C. D. (1982). Theorie und Messung der emotionalen und kognitiven Komponente der Prüfungsangst [Theory and measurement of the emotional and cognitive components of test anxiety]. Zeitschrift für Differentielle und Diagnostische, 3(3), 169-184.

IBM. (2013). IBM SPSS Statistics, Version 22. Chicago: Author.

Kavanagh, B. E., Ziino, S. A., \& Mesagno, C. (2016). A comparative investigation of test anxiety, coping strategies, and perfectionism between Australian and United States students. North American Journal of Psychology, 18(3), 555-570.

Knappe, S., Beesdo-Baum, K., Fehm, L., Stein, M. B., Lieb, R., \& Wittchen, H. U. (2011). Social fear and social phobias types among community youth: Differential clinical features and vulnerability factors. Journal of Psychiatric Research, 45, 111-120. https://doi.org/10.1016/j.jpsychires.2010.05.001

Little, T. D. (2013). Longitudinal structural equation modeling. New York: Guilford Press.

Lowe, P. A. (2018a). An investigation into the psychometric properties of the Test Anxiety Measure for College Students. Journal of Psychoeducational Assessment, 36, 322-336. https://doi.org/10.1177/0734282916678536

Lowe, P.A. (2018b). The Test Anxiety Measure for College Students: Examination of its psychometric properties using an online survey with a Canadian sample. Canadian Journal of School Psychology, 33, 279-296. https://doi.org/10.1177/0734282017724904

Lowe, P.A. (2019a). Cross-national comparison between U.K. and U.S. higher education students in test anxiety. Higher Education Studies, 9, 88-97. https://doi.org/doi:10.5539/hes.v9n3p88

Lowe, P. A. (2019b). Exploring cross-cultural and gender differences in test anxiety among U.S. and Canadian college students. Journal of Psychoeducational Assessment, 37, 112-118.

Lowe, P. A., \& Lee, S. W. (2008a). Factor structure of the Test Anxiety Inventory for Children and Adolescents (TAICA) scores across gender among students in elementary and secondary school settings. Journal of Psychoeducational Assessment, 26, 231-246. https://doi.org/10.1177/0734282907303773

Lowe, P. A., Lee, S. W., Witteborg, K. M., Pritchard, K. W., Luhr, M. E., Cullinan, C. M., ... Janik, M. (2008b). The Test Anxiety Inventory for Children and Adolescents (TAICA). Examination of the psychometric properties of a new multidimensional measure of test anxiety among elementary and secondary school students. Journal of Psychoeducational Assessment, 26, 215-230. https://doi.org/10.1177/0734282907303760

Mills, J. S., \& Blankstein, K. R. (2000). Perfectionism, intrinsic vs extrinsic motivation, and motivated strategies for learning: A multidimensional analysis of university students. Personality and Individual Differences, 29, 1191-1204. https://doi.org/10.1016/S01918869(00)00003-9

Mowbray, T., Boyle, C., \& Jacobs, T. (2015). Impact of item orientation on the structural validity of self measures: An investigation using the TAI-G in an Australian sample. Journal of Psychoeducational Assessment, 33, 278-290. https://doi.org/10.1177/0734282914548405

Muthén, L. K., \& Muthén, B. O. (1998-2013). Mplus Version 7.11. Los Angeles, CA: Muthén \& Muthén.

Ollendick, T. H., King, N., \& Frary, R. B. (1989). Fears in children and adolescents: Reliability and generalizability across gender, age and nationality. Behaviour Research and Therapy, 27, 19-26. https://doi.org/10.1016/0005-7967(89)90115-0

Onwuegbuzie, A. J., \& Daley, C. E. (1999). Perfectionism and statistics anxiety. Personality and Individual Differences, 26, 1089-1102. https://doi.org/10.1016/S0191-8869(98)00214-1

Rafferty, B. D., Smith, R. E., \& Ptacek, J. T. (1997). Facilitating and debilitating trait anxiety, situational anxiety, and coping with an anticipated stressor: A process analysis. Journal of Personality and Social Psychology, 72, 892-906. https://doi.org/10.1037/0022-3514.72.4.892

Reiss, N., Warnecke, I., Tolgou, T., Krampen, D., Luka-Krausgrill, U., \& Rohrmann, S. (2017). Effects of cognitive behavioral therapy with relaxation vs. imagery rescripting on test anxiety: A randomized controlled trial. Journal of Affective Disorders, 208, 483-489. https://doi.org/10.1016/j.jad.2016.10.039 
Russell, A., Hart, C. H., Robinson, C. C., \& Olsen, S. F. (2003). Children's sociable and aggressive behaviour with peers: A comparison of the US and Australia, and contributions of temperament and parenting styles. Journal of Behavioural Development, 27, 74-86. https://doi.org/10.1080/0165025244000038

Sarason, I. G. (1984). Stress, anxiety, and cognitive interference: Reactions to Tests. Journal of Personality and Social Psychology, 46, 929-938. https://doi.org/10.1037/0022-3514.46.4.929

Spielberger, C. D. (1980a). Test Anxiety Inventory. Palo Alto, Consulting Psychologists Press.

Spielberger, C. D. (1980b). Test Anxiety Inventory: Manual. Palo Alto, Consulting Psychologists Press.

Stöeber, J., Feast, A. R., \& Hayward, J. A. (2009). Self-oriented and socially prescribed perfectionism: Differential relationships with intrinsic and extrinsic motivation and test anxiety. Personality and Individual Differences, 47, 423-428. https://doi.org/10.1016/j.paid.2009.04.014

Tatum, T., Lundervold, D. A., \& Ament, P. (2006). Abbreviated upright Behavioral Relaxation Training for test anxiety among college students: Initial results. International Journal of Behavioral Consultation and Therapy, 2, 475-480. https://doi.org/10.1037/h0101001

Taylor, J. T. (1956). Drive theory and manifest anxiety. Psychological Bulletin, 53, 303-328. https://doi.org/10.1037/h0040353

Thomas, C. L., \& Cassady, J. C., \& Heller, M. L. (2017). The influence of emotional intelligence, cognitive test anxiety, and coping strategies on undergraduate academic performance. Learning and Individual Differences, 55, 40-48. https://doi.org/j.lindif.2017.03.001

Triventi, M. (2014). Higher education regimes: An empirical classification of higher education systems and its relationship with student accessibility. Quality and Quantity, 48, 1685-1703. https://doi.org/10.1007/s11135-013-9868-7

Yang, Y., \& Green, S. B. (2011). Coefficient alpha: A reliability coefficient for the 21st century? Journal of Psychoeducational Assessment, 29, 377-392. https://doi.org/10.1177/0734282911406668

Zeidner, M. (1998). Test anxiety: The state of the art. New York: Plenum Pre

\section{Copyrights}

Copyright for this article is retained by the author(s), with first publication rights granted to the journal.

This is an open-access article distributed under the terms and conditions of the Creative Commons Attribution license (http://creativecommons.org/licenses/by/4.0/). 